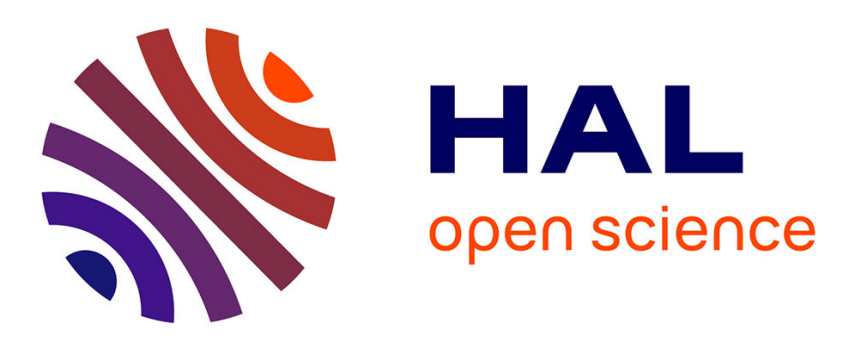

\title{
Ethos préalable et éthos (re)construit. La transformation de l'humour légendaire de François Hollande
}

Anna Jaubert, Damon Mayaffre

\section{- To cite this version:}

Anna Jaubert, Damon Mayaffre. Ethos préalable et éthos (re)construit. La transformation de l'humour légendaire de François Hollande. Langage et Société, 2013. hal-01978523

\section{HAL Id: hal-01978523 \\ https://hal.science/hal-01978523}

Submitted on 21 Jan 2019

HAL is a multi-disciplinary open access archive for the deposit and dissemination of scientific research documents, whether they are published or not. The documents may come from teaching and research institutions in France or abroad, or from public or private research centers.
L'archive ouverte pluridisciplinaire HAL, est destinée au dépôt et à la diffusion de documents scientifiques de niveau recherche, publiés ou non, émanant des établissements d'enseignement et de recherche français ou étrangers, des laboratoires publics ou privés. 


\section{ETHOS PRÉALABLE ET ETHOS (RE) CONSTRUIT LA TRANSFORMATION DE L'HUMOUR LÉGENDAIRE DE FRANÇOIS HOLLANDE}

Anna Jaubert et Damon Mayaffre

Éditions de la Maison des sciences de l'homme | «Langage et société »

2013/4 N 146 | pages 71 à 88

ISSN 0181-4095

ISBN 9782735116072

Article disponible en ligne à l'adresse :

https://www.cairn.info/revue-langage-et-societe-2013-4-page-71.htm

Distribution électronique Cairn.info pour Éditions de la Maison des sciences de l'homme.

(C) Éditions de la Maison des sciences de l'homme. Tous droits réservés pour tous pays.

La reproduction ou représentation de cet article, notamment par photocopie, n'est autorisée que dans les limites des conditions générales d'utilisation du site ou, le cas échéant, des conditions générales de la licence souscrite par votre établissement. Toute autre reproduction ou représentation, en tout ou partie, sous quelque forme et de quelque manière que ce soit, est interdite sauf accord préalable et écrit de l'éditeur, en dehors des cas prévus par la législation en vigueur en France. Il est précisé que son stockage dans une base de données est également interdit. 


\title{
Ethos préalable et ethos (re)construit La transformation de l'humour légendaire de François Hollande
}

\author{
Anna Jaubert \\ UMR Bases, Corpus, Langage (CNRS - Université de Nice Sophia \\ Antipolis), ajaubert@unice.fr \\ Damon Mayaffre \\ UMR Bases, Corpus, Langage (CNRS - Université de Nice Sophia \\ Antipolis), mayaffre@unice.fr
}

\section{Introduction. Ethos et rhétorique}

$\mathrm{Si}$ un discours se doit convaincant, c'est bien celui d'une campagne électorale, mais l'impérieuse vocation à remporter un maximum de suffrages dépasse de loin une construction purement argumentative. C'est qu'un discours électoral ne s'adresse pas à la seule raison des électeurs; émotions, identification, passions, y ont une large part: avec lui, et plus que jamais, convaincre c'est persuader, et persuader c'est d'abord séduire. Pour un candidat, mettre les rieurs de son côté, disqualifier un adversaire par la dérision, sont des stratégies de bonne guerre: les commentateurs politiques le savent, mais l'impact de ces stratégies est aussi amplifié par ces mêmes commentateurs qui dans le débat font la part belle à la " petite phrase ", au trait d'esprit. On ne peut apprécier la valeur humoristique d'un discours indépendamment de ses conditions de production et de réception. La caisse de résonance offerte par les médias, et le trope communicationnel $^{1}$ qui fait du public le destinataire principal dont on suppute les attentes, jouent ici un rôle déterminant.

1. La notion de trope communicationnel développée par (Kerbrat-Orecchioni, 1986) s'applique à des situations de communication où ceux qui assistent en tiers à une interaction verbale sont en réalité les premiers à qui le discours s'adresse: c'est le cas des débats et interviews télévisés ou radiodiffusés. 
Or il se trouve que dans la campagne des élections présidentielles de 2012, le candidat François Hollande connu depuis longtemps pour son humour, en a usé avec une modération remarquable afin de construire une nouvelle image de lui-même, celle d'un présidentiable précisément ${ }^{2}$, et non plus celle d'un chef de parti ou d'un élu de la Corrèze. Rappelons que l'argumentation électorale est sous-tendue par son enjeu, la demande de confiance qu'exige la fonction briguée. De ce fait, plus que jamais, le discours argumentatif se fond dans un discours éthique, entendons cet adjectif littéralement: un discours expressément construit pour projeter un ethos du locuteur. En l'occurrence un ethos qui le recommande à son auditoire ${ }^{3}$, et tente d'assurer la place postulée ${ }^{4}$. L'efficacité d'un discours passe par la crédibilité de celui qui parle (Aristote 1991, I: 22-23), c'est sur cet impératif que se fondaient les conseils prodigués aux orateurs dans l'Antiquité: la rhétorique et la politique ont toujours fait bon ménage.

Le discours politique, qui souvent se prête avec succès à l'analyse logométrique (voir Mayaffre, 2005, 2012a et 2012b), ne peut compter ici sur la démarche informatisée. C'est que l'humour et l'ironie sont des comportements énonciatifs (de double jeu, d'ambiguïté) : ils ne sont pas isolables sur des segments que l'on pourrait dénombrer. Leur identification, et leur efficience d'actes de langage, requiert l'imprégnation d'un interdiscours ${ }^{5}$, et une compétence encyclopédique partagée où s'impose notamment la mémoire des discours politiques antérieurs. Le plein rendement stratégique des traits d'esprit, ironiques ou humoristiques, n'est atteint qu'à ce prix. Dans les pages qui suivent, nous observerons quelques séquences significatives, engageant une analyse pragmatique de

2. Dans son Petit Hollande illustré par l'exemple, Jouan (2012: 7-8) se fait l'écho d'un constat général: " Dans la geste gaullienne, on ne peut rêver de devenir président sans faire un travail sur soi-même [...] Alors quand François Hollande a pris la décision de se lancer dans la bataille présidentielle [et qu'] il officialise son entrée en lice dans la primaire socialiste le 31 mars à Tulle, il s'est fait deux promesses: perdre sa mauvaise graisse... et son humour ".

3. Pour une réflexion sur l'ethos, ou image de soi dans le discours, et son impact argumentatif, voir Amossy (1999, 2006, 2010).

4. Tout discours véhicule implicitement une demande de reconnaissance de place, " parole intermédiaire ", qui fait entendre un "Qui-je suis pour toi/qui tu es pour moi » ou plutôt "qui je veux être pour toi, qui je veux que tu sois pour moi " (Flahault, 1979). Cette demande est cruciale dans un discours de campagne.

5. Au sens large " on appelle 'interdiscours' l'ensemble des unités discursives (relevant de discours antérieurs du même genre, de discours contemporains d'autres genres, etc.) avec lesquelles un discours particulier entre en relation implicite ou explicite " (Charaudeau \& Maingueneau dirs, 2002 : 324). 
ces traits, considérés comme des figures macrostructurales ${ }^{6}$ qu’il convient $^{\prime}$ de distinguer. Ce parti impose d'articuler leur manifestation aux données contextuelles qui les motivent et les conditionnent.

Nous mettrons d'abord en perspective le caractère traditionnel de la dérision dans le champ du discours politique (1); nous en examinerons ensuite l'usage par le candidat Hollande en situation nouvelle: la course à l'Élysée, et son indispensable différenciation par rapport au président sortant (2). D'un humour désormais sous surveillance, enrôlé dans une " guerre des ethê ", nous tirerons quelques enseignements sur la question d'un bon réglage de la plaisanterie, sur le contrôle de ses effets, et au final sur la maîtrise d'un exercice rhétorique sans doute plus contraint qu'on le croit (3).

\section{Discours politique et dérision}

\subsection{Une dialectique des registres. Exemples historiques}

Le discours politique se signale classiquement par une dialectique des registres. Alors que s'impose massivement la sacralisation ${ }^{7}$ des sujets traités (la nation, le peuple, la crise, etc.), motivant un ton grave, et que parfois même l'argumentation dramatisée tourne à l'ultimatum (" moi ou le chaos "), on observe aussi, et comme une salutaire respiration, la tendance inverse: des épisodes discursifs désacralisants, portés par la dérision ou par l'autodérision. De fait la désacralisation, le dérisoire, l'humour sont le plus souvent une arme de l'homme politique à bout d'argument. Les exemples sont nombreux dans l'histoire française ${ }^{8}$; un des plus fameux est sans doute celui de De Gaulle lors des premières élections présidentielles au suffrage universel. À trois jours du scrutin, et alors que contre toute attente de sa part, il est en ballottage, contesté pour sa rigidité nationaliste, le voilà à l'écran en train de mimer ses propos:

Bien entendu, on peut sauter sur sa chaise comme un cabri en disant l'Europe! l'Europe! l'Europe! mais cela n'aboutit à rien et cela ne signifie rien. Entretien télévisé, 14 décembre 1965.

En joignant une gestuelle burlesque à la parole, le grand homme jouait certes avec son image, mais pour immédiatement en communiquer le ridicule aux européistes. La désacralisation de sa personne affiche une transgression posturale qui éclabousse ceux qu'elle dénonce: décrédibilisant la source de la critique, dont on ne retient que l'ethos gesticulateur, de Gaulle

6. Pour une approche pragmatique des figures du discours, voir Bonhomme (2005, 2013) et Jaubert $(2008,2013)$.

7. Pour le rapport entre le politique et le sacré, voir par exemple Guyonnet (1997).

8. Pour le traitement de nombreux exemples non français, voir Tsakona \& Popa (2012). 
produisait une pirouette argumentative propre à vider l'abcès de fixation. Plus éloigné de nous, un autre grand homme fit un choix analogue, celui de jouer avec son image plutôt que de répondre sur le fond. Clémenceau, le Tigre, accusé devant l'Assemblée par un jeune député socialiste de trahir ses convictions politiques passées et d'être devenu "un bourgeois cossu " eut la répartie spirituelle "Je vous remercie pour la cédille». Un humour à multiples rebonds: ouf, il s'en fallait de peu que l'accusation soit plus désagréable! mais la dérision n'est pas seulement divertissante (même au sens initial de "faisant diversion »), elle s'adjoint une contre-attaque, car si la faute d'orthographe ("* *oçu ») est prêtable à l'opposant, celui-ci n'est plus qu'un âne. Ecorner l'ethos de l'accusateur rend une position haute à l'accusé qui affecte d'ignorer le coup porté.

Pour autant il s'agit là de points marqués de façon conjoncturelle, d'un avantage pris sur l'adversaire très provisoirement. C'est pourquoi toutes les postures, humoristiques ou ironiques, entrent en négociation avec des données factuelles: statut institutionnel, position dans les sondages, antériorité de l'image publique, c'est-à-dire de l'ethos préalable'. Dans un tel contexte, l'humour est plutôt défensif, visant à dédramatiser l'interaction, à minorer la portée d'une attaque, alors que l'ironie pour sa part, est clairement offensive. Les notions de sous-énonciation vs surénonciation que l'on doit à Alain Rabatel (2013) permettent de raisonner le choix des armes.

\subsection{Une offensive ironique dans la campagne 2012}

La différence entre l'ironie et l'humour doit être ici explicitée. Si l'on peut les hiérarchiser, à l'instar de Charaudeau (2006 et 2011) qui fait de l'humour, ou plus exactement des "catégories humoristiques", une notion englobante, on peut aussi considérer qu'il s'agit d'actes de langage distincts qui, ancrés tous deux dans la duplicité énonciative et le faire-semblant, construisent chacun un ethos différent. A. Rabatel oppose, à juste titre selon nous, "la raillerie clivante de l'ironie " à "la moquerie complice de l'humour" (Rabatel, 2013). Son approche dégage le principe de leur divergence pragmatique, essentiellement une posture de "sur-énonciation " pour la première, caractérisée par le rejet radical de l'autre énonciation mise en scène, et de "sous-énonciation " pour la seconde qui joue plutôt avec l'altérité.

9. La notion d'ethos préalable renvoie au prédiscursif. Elle est adoptée par plusieurs analystes du discours (Adam, 1999, Amossy, 1999, 2000, 2010, Maingueneau, 1999). Avec raison si l'on considère que l'ethos préalable constitue, comme nous le montrerons, une détermination situationnelle incontournable pour la construction de l'ethos discursif. 
Le discours de campagne de Besançon prononcé par Nicolas Sarkozy le 30 mars 2012, illustre pour sa part la stratégie ironique, qui en l'occurrence procède par assauts réitérés (d'où la nécessité ici d'en transcrire un large extrait):

Les sujets qui intéressent Monsieur Hollande... Là on a été gâté, il faut bien le dire [pause et rires du public]. Il y a quelque chose qui vous troublait, mes chers compatriotes, depuis longtemps, c'est la présence du mot race dans le préambule de 1946 de la constitution [silence, mimique narquoise, et rires du public]. Moi qui depuis 5 ans, parcours la France dans tous les sens, je dois avouer que je suis sans arrêt harcelé par des gens qui, me promenant, me disent « monsieur le Président, c'est un scandale, arrêtez avec le mot race dans le préambule de 1946 de la Constitution! » [rires du public]. Et voilà que monsieur Hollande a trouvé une idée fantastique: pour supprimer le racisme supprimons le mot race. [pause et applaudissements]. Comme c'est un homme qui a de la suite dans les idées, pour supprimer les injustices, il dit "supprimons le mot zone " [pause de l'orateur et rires]. Mais il manque d'ambition! Pendant qu'on y est, il faut supprimer le mot " pauvre ". Et pour résoudre le problème du chômage supprimer le mot « chômeur ». On serait tranquille! [pause de l'orateur et applaudissements] Et ça veut gouverner la France! [...]

Alors continuons sur ses propositions. Figurez-vous qu'il y a un problème: le prix du pétrole augmente! [...] Il a donc eu une idée remarquable [pause ironique renforcée de mimiques]. On va bloquer les prix du pétrole! [nouvelle pause de l'orateur et rires du public] Et là, on voit déjà les Saoudiens, les Iraniens, les Irakiens trembler devant la perspective! On bloque le prix du pétrole. [pause de l'orateur] Ça fait peur! [rires et applaudissements] Et ça veut diriger la France! [...]

Voilà que la priorité c'est de démolir la filière nucléaire. [...] Ah oui, mais nous dit-il, tout a changé avec Fukushima. Eh ben réfléchissons. [...] Mais là où je n'ai pas suivi le raisonnement de Monsieur Hollande c'est qu'il a dit après Fukushima "la première centrale à fermer c'est Fessenheim " [très long silence peuplé de mimiques dub itatives et ironiques de l'orateur, et manifestations du public]. Alors je me suis précipité sur un livre de géographie [pause de l'orateur et rires du public], car ça me semblait si énorme [pause de l'orateur et applaudissements du public] que je me suis dit " c'est moi qui dois faire une erreur " [pause de l'orateur et applaudissements du public]. Fessenheim: l'Alsace [silence ironique] : la plage est assez éloignée! [rires et applaudissements] Et ça veut diriger la France! [applaudissements du public]. La semaine dernière, j'ai été visité une autre centrale qu'il veut absolument fermer: St Laurent-les-eaux [silence ironique et mimiques de l'orateur]. Je me suis dit: « là, ils n'ont quand même pas refait le coup. [rires du public] Ils ne se sont quand même pas retrompés, c'est pas possible ». Je vais donc confiant à St Laurent-les-eaux et je vois La Loire [rire de l'orateur et du public]. Et ça veut diriger la France!

Discours de Nicolas Sarkozy à Besançon, 30 mars 2012. 
Pour éreinter, ou disons-le plus familièrement, pour casser l'image de son adversaire, Nicolas Sarkozy caricature ses propositions. Une des plus fréquentes caractéristiques de l'ironie, on le sait bien, c'est le recours à la feinte naïveté ${ }^{10}:$ l'énonciateur fait semblant d'être incrédule devant tant de bêtise. La feinte est ici mise en scène de façon très appuyée (à grand renfort de pauses et de mimiques) et le discours vire au persiflage ${ }^{11}$, ponctué par sa glose, l'anaphore méprisante «Et ça veut diriger la France! ». Mais sans doute l'orateur a-t-il ici passé la mesure: mépriser explicitement un adversaire, lui dénier le statut humain (par le pronom neutre ça) peut avoir un effet boomerang. Dans le traitement des "faces", les images projetées interagissent. À trop dégrader l'autre, on peut se dégrader soimême. Le jeu de massacre ironique, qui est censé gagner les rieurs à sa cause, s'abaisse à trop quêter la connivence, à " en rajouter ", comme dans un show qui ne recueillerait pas spontanément tous les applaudissements espérés ${ }^{12}$.

L'humour pour sa part est une arme moins lourde à manier mais plus délicate aussi, car il repose souvent sur des positionnements complexes.

\section{La figure du présidentiable. Le nouvel ethos de François Hollande}

\subsection{L'homme public et sa transformation}

Partons d'un constat: dans le livre programmatique de François Hollande, Changer de destin ${ }^{13}$, la moisson des traits d'esprit s'avère plutôt maigre. L'humour légendaire de l'homme a été mis en veilleuse ${ }^{14}$, et cela n'a rien de surprenant. L'heure n'est plus à la plaisanterie, celui qui, à la suite des primaires du parti socialiste, vient d'être intronisé candidat doit, dans et par son discours, confirmer son image de présidentiable, plus sobre à tous

10. Voir Berrendonner (2002) et Jaubert (2013). Au-delà de l'antiphrase, l'ironie émerge en contexte de procédures diverses marquées par la duplicité énonciative (Jaubert, 2009).

11. L'acte de langage ironique offre des nuances: selon son accompagnement mimo-gestuel et la situation d'énonciation, il dose ses effets entre critique ludique, spirituelle, et sarcasme caractérisé (par l'exagération caricaturale de la négativité). Nous définirons le persiflage comme un acte ironique insistant faisant volontiers appel au sarcasme. Pour une réflexion sur la différence entre sarcasme et ironie, voir Vivero García (2011 : 46-49).

12. La vidéo du meeting de Besançon témoigne d'une salle rieuse certes, mais légèrement mal à l'aise: dans les meetings suivants, Sarkozy va tenter d'améliorer sa performance (il supprime notamment la scansion «Et ça veut gouverner la France!»). Cf. le meeting de Caen, 6 avril 2012.

13. F. Hollande, Changer de destin, Paris, Robert Laffont, 2012.

14. On note d'ailleurs que le recueil de plaisanteries de Jouan (2012) se nourrit surtout d'exemples puisés dans une période antérieure à la campagne présidentielle. 
points de vue ${ }^{15}$ et capable de se hausser au niveau de gravité attendu d'un chef d'état. Significativement l'ethos sérieux est le premier mentionné par Charaudeau (2005 : 91-96) dans la panoplie des " ethos de crédibilité »: il s'impose par les responsabilités et le caractère régalien de la fonction.

Les rares traits d'esprit que l'on relève vont dans ce sens. Ce sont des exemples d'humour très médité, taillé sur mesure pour construire le nouvel ethos visé. Si dès les premiers chapitres il est bon d'assumer son ambition, avec des titres comme « Être soi-même » ou "Ma décision », il est aussi judicieux d'afficher le recul d'une saine autodérision. Après s'être présenté brièvement, et avoir déclaré, non sans fierté, vouloir mettre ses pas dans ceux de ses grands devanciers, citant de Gaulle et faisant l'éloge de Mitterrand, François Hollande revient sur ses fonctions de premier secrétaire, sur l'abandon de ce poste en 2008, et sur son repli en Corrèze. Mais l'autobiographie pourrait verser dans l'auto-hagiographie, n'était un subtil bémol:

La traversée, non du désert mais du plateau des Millevaches, m’a été précieuse.

Changer de destin, page 17.

Sacralisation et désacralisation: la formule est habile. Elle renvoie au rite initiatique qui forge son grand homme, "la traversée du désert ". L'image originellement collée au général de Gaulle (1946-1958), a été reprise pratiquement par tous: Mitterrand après ses multiples défaites, Chirac entre 1993 et 1995 avec son isolement hors du gouvernement et la trahison de Balladur; ou encore par Nicolas Sarkozy pour qualifier sa mise en quarantaine dans la période 1995-2002 par le clan Chirac. Mais la prétérition, qui substitue au noble désert le rustique plateau des Millevaches, décline la référence sur le mode mineur, le rapprochement glorieux est tempéré par cet humour qui convoque le mot "vache ", et avec lui le plateau... de la "normalité ".

Entre bienveillance et causticité, c'est encore par un cocktail tonal qu'il accrédite l'ethos de la modération, qui est aussi celui d'une inévitable ambiguiité, car pour Hollande, disqualifier son concurrent François Bayrou relève d'une double contrainte ${ }^{16}$. L'ironie affecte une certaine tempérance:

15. On se souvient que cette transformation, concertée et assumée, a commencé par une cure d'amaigrissement relevée par les médias, et que l'ethos sobre s'est affirmé dans la stratégie discursive des primaires, F. Hollande proposant un discours lisse (potentiellement rassembleur), en retrait manifeste par rapport à celui de ses concurrents.

16. Il s'agit d'écorner son image suffisamment pour l'écarter d'un second tour (dont, rappelons-le, plusieurs sondages l'annonçaient vainqueur quel que soit son adversaire), tout en ménageant la possibilité d'un ralliement final. 
Reste François Bayrou, cet intrépide chevalier de la petite escouade centriste. C'est un admirateur d'Henri IV, ce qui prouve qu'il ne peut être entièrement mauvais. Suivez mon panache, dit-il. Mais c'est un panache gris. Son programme est un anti-programme. Comme si ne rien promettre résumait une politique. En le lisant, je suis saisi par la peur du vide. Ibid., page 132-133.

Verse-t-elle pour autant dans la posture connivente de l'humour? Non, le propos reste bien ironique car la cible est clairement l'autre: François Hollande fait de l'esprit aux dépens de François Bayrou. Ainsi l'exagération burlesque, " je suis saisi par la peur du vide » qui vient ponctuer l'accusation d'inconsistance, couvre à peine du vernis ludique de la plaisanterie une ultime estocade. Dire en riant, ce n'est pas dire " pour rire ", et c'est dire quand même. Par ailleurs, si l'attaque ironique s'est voulue mâtinée de quelques concessions valorisantes, ces dernières sont vite balayées: l'intrépide chevalier n'a pour lui qu'une " petite escouade ", et son panache est terne. Mais on mesure ici la subtilité du procédé: une ironie bridée suffit; en soi le refus de l'artillerie lourde déclasse l'adversaire au rang de simple comparse.

La plaisanterie désormais très contrôlée, soucieuse de proportionner ses attaques, dessine la figure d'un homme réfléchi, maître de lui et de son discours, animé « d'une certaine idée » du présidentiable.

\subsection{La guerre des ethê}

L'ethos du discours de campagne est ainsi appelé à remplacer l'ethos préalable du candidat. L'enjeu est de taille pour lui, car Nicolas Sarkozy et son camp arguent d'un défaut d'expérience, voire de légitimité: Hollande n'a jamais été ministre! Qu'à cela ne tienne, puisque le président sortant a déçu les électeurs, notamment par les excès de l'attitude " décomplexée », le prétendant a tout à gagner d'une figure alternative.

L'ethos que François Hollande s'emploie à promouvoir est donc un ethos a contrario. Non seulement par rapport à sa personnalité antérieure, mais aussi par rapport à celle de l'adversaire en place. Il sera certes plus grave, et donc moins enclin à manier l'humour que le François Hollande jusque-là connu, mais il sera surtout modéré et pacificateur là où Nicolas Sarkozy fait valoir son caractère de " battant ». N'oublions pas que le fond $\mathrm{du}$ discours se déclare aussi dans sa forme. Celui qui dit en substance: "moi, je ne veux pas diviser les Français, je ne veux pas les opposer les uns aux autres ", doit savoir dans le feu de l'action retenir ses coups et, ce qui est plus utile encore, montrer qu'il les retient, qu'un intérêt supérieur l'habite et qu'il conserve son sang-froid ${ }^{17}$.

17. P. Moscovici rappelait à l'antenne de France 2, dimanche 3 février 2013, qu'en tant que directeur de campagne de Hollande (et ami de 30 ans), il avait été à même de 
La mémoire de l'ethos préalable est alors précieuse, et il convient de la raviver à bon escient. Rappelant ses dispositions naturelles, F. Hollande fait d'un trait ironique deux coups pour récupérer à son avantage le reproche de n'avoir jamais été ministre: cela tombe plutôt bien pour quelqu'un qui entend faire autrement, et combien de fois d'ailleurs a-t-on entendu le reproche inverse adressé à ceux qui dans le passé avaient été plusieurs fois ministres... Il faudrait savoir! Livré sur le mode plaisant, l'argument n'en brille pas moins par sa logique implacable, il emblématise l'ouverture de la campagne, dans ce discours du Bourget, où F. Hollande a publiquement «fendu l'armure »:

Certains me reprochent de n'avoir jamais été ministre. Quand je vois ceux qui le sont aujourd'hui, ça me rassure! Et ce sont les mêmes qui reprochaient en son temps à François Mitterrand de l'avoir été onze fois. Et dois-je rappeler, en gardant la comparaison, que Georges Clémenceau ne devint ministre et président du Conseil qu’à 65 ans? Mais je n’attendrai pas jusque-là, je vous le promets.

Discours prononcé au Bourget, le 22 janvier 2012.

La défense et l'attaque sont ici indissociables: le "manque " allégué devient un mauvais procès intenté par des esprits incohérents. Au moment choisi, et précisément parce qu'il n'est plus galvaudé, l'humour de Hollande peut devenir une force de frappe. Cela va se confirmer.

Dans le face-à-face télévisé entre les deux candidats, appelé significativement duel, le fleuret pourrait bien ne pas être moucheté. L'expression du visage parle elle aussi. François Hollande, légèrement souriant et, ne l'oublions pas, en situation favorable dans les sondages, semble en embuscade: il n'a pas sorti l'arme de la dérision, mais on l'en sait capable, et le possible pèse comme une menace. Finalement, sous la pression, c'est Nicolas Sarkozy qui rappelle l'ethos préalable de son adversaire, censé le disqualifier:

Sarkozy: ... quel est le pays, le pays, il y a en un, qui n'a pas connu la récession depuis 2009? C'est la France.

Hollande: ... c'est marrant... vous êtes toujours content de vous... C'est extraordinaire. Quoi qu'il arrive, quoi qu'il se passe, vous êtes content... Les Français le sont moins, mais vous, vous êtes content...

Sarkozy: Monsieur Hollande, dois-je considérer que lorsque vous mentez de façon éhontée, je dois accepter?

Hollande: Je n'ai rien dit qui puisse justifier cette expression...

Sarkozy: C'est un mensonge!

Hollande: Lequel?

Sarkozy: C’est un mensonge!

relever les qualités d'un président en puissance, parmi lesquelles la capacité à rester froid, disait-il, « au bon sens du terme ». 
Hollande: Lequel?

Sarkozy: C'est un mensonge!

Hollande: Lequel?

Sarkozy: ... lorsque vous dites que je suis toujours content... C'est un mensonge!

Hollande: Ah bon? Donc vous êtes très mécontent de vous. J'ai dû me tromper, j'ai dû faire une erreur. Je me mets à présenter mes excuses...

Sarkozy: ... ce n'est pas le concours de la petite blague...

Hollande: ...vous êtes très mécontent de vous

Sarkozy : ...monsieur Hollande, ce n'est pas le concours de la petite blague...

Hollande: ... non, non, ce n'est pas la petite blague...

Débat, François Hollande/Nicolas Sarkozy, 2 mai 2012 ${ }^{18}$.

Souvenir évident du fameux surnom "Monsieur petite blague »: l'image est effectivement peu présidentielle, mais nous sommes en fin de campagne, et, depuis des mois, tout le nouveau discours de Hollande s'est employé à la corriger. Avec succès semble-t-il : l'allusion de Sarkozy tombe en porte à faux, elle est impuissante à le sortir du piège verbal qu'il a refermé sur lui-même en dégainant trop vite, une accusation solennelle disproportionnée: "C'est un mensonge! » qui, dans le champ clos de la joute verbale, réduit à une alternative la marge des reprises ${ }^{19}$. Or l'aveu de satisfaction comme celui d'insatisfaction sont pour un président sortant aussi impossibles l'un que l'autre. La dérision, on le constate, veut que la balle soit placée sur le bon terrain, elle exige une parfaite maîtrise du contexte, et aussi du cotexte, c'est-à-dire en l'occurrence de l'enchaînement possible des réparties.

\section{Congruence de I'humour}

\subsection{Question de face, question de place}

Le changement d'ethos construit par les discours de campagne de F. Hollande, met en évidence une palette de stratégies humoristiques qui nuancent le clivage admis entre humour et ironie ${ }^{20}$. Nous avons rappelé supra la pirouette argumentative que l'humour pouvait constituer, et son rôle de refuge pour l'homme politique à bout d'argument, ou qui du moins n'a pas intérêt à entrer dans le vif d'un sujet qui fâche. Observons maintenant sa détermination en amont: mû par la même

18. $37^{\mathrm{e}}$ minute et suivantes du débat, consultable sur dailymotion.com ou youtube.com.

19. Contrairement à un " vous exagérez " qui aurait permis de réorienter l'interaction en contre-attaquant sur un défaut de nuance.

20. Rabatel (2013) les distingue en liant la gerbe des paramètres divergents intuitivement reconnus; mais ces divergences ne s'actualisent pas toujours en totalité rendant l'opposition parfois poreuse. 
nécessité de résoudre un embarras, et produit par le même homme, l'humour change avec son statut institutionnel.

Alors que le candidat Hollande ne peut ni taire ni approfondir le dissensus qui existe, et qui est loin d'être anodin, entre sa vision d'une politique énergétique et celle des Verts, ses alliés souvent turbulents, il se permet d'écarter le problème à la faveur d'un jeu de mots facile:

C'est une alliance [sur l'essentiel] qui vaut la peine d'un accord, en oubliant l'accroc [sur le nucléaire]!

Changer de destin, p. 132.

En matière de trait d'esprit, le calembour n'a pas toujours eu très bonne réputation en France, et si son image se trouve aujourd'hui réhabilitée par la pratique journalistique, c'est parce qu'il habille le propos d'une connotation ludique ${ }^{21}$, détachée: si " accord » vaut « accroc » au motif d'une simple ressemblance phonique, la divergence est renvoyée à l'épiphénomène sans conséquence qu'on voudrait pieusement qu'elle soit. Mais les faits sont têtus, et ce procédé n'est guère à la hauteur de l'ethos crédible que le candidat en campagne allait ensuite promouvoir.

Élu Président, et lors d'une première conférence de presse, F. Hollande est confronté aux journalistes qui se flattent, comme souvent, de pousser l'homme politique dans ses derniers retranchements, insistant pour obtenir un mea culpa sur ses six premiers mois d'exercice du pouvoir. L'embarras relève ici d'une menace immédiate pour son image ${ }^{22}$ : l'interpellé doit le résoudre à chaud et clore la séance en reprenant un avantage de position. C'est un humour désormais « mitterrandisé " qui offre à Hollande une porte de sortie:

Sur la question des erreurs, tout à l'heure, il m'était rappelé toutes les Unes de magazine. Dans tout ce qui a été dit, j’imagine qu'il doit y avoir une part qui répond à votre question. Si en plus il faut que je fasse ici mon autocritique, mais ce serait accablant! Je ne veux pas prendre ce risque. Mais si l'erreur, la seule que j'aurais pu commettre, était de vous avoir invité à venir ici à l'Élysée pour cette conférence de presse, je la reconnais et je demande ici grâce et pardon. Merci.

Première conférence de presse, mardi 13 novembre 2012.

21. Voir Jaubert 2011.

22. Par rapport à l'exemple précédent trois paramètres en fait ont changé: non seulement le statut du locuteur (candidat $v$ Président de la République), déterminant pour un rapport de place, mais aussi la situation d'énonciation (un livre $v s$ une interaction in praesentia) et la cause de l'embarras (une question de programme $v s$ une question d'image). 
Le voile des formules concessives ("j’imagine qu'il doit y avoir une part qui répond..." ", si l'erreur, la seule que j'aurais pu commettre...») esquisse, mais faiblement, l'indécidabilité illocutoire de l'humour: le minimum requis pour une fin de non-recevoir élégante, assortie de la remise en place qui sied à un Président de la République. Pareille stratégie doit sans doute être rapportée à la culture française. L'appréciation de l'humour comme de l'ironie est affaire de public, la réception est conditionnée par un imaginaire collectif où le savoir-vivre conversationnel d'un homme du monde (" mais ce serait accablant»!), et l'image discrètement hautaine d'un " monarque républicain " jouissent d'une certaine cote.

En réalité l'exercice de l'humour en politique s'avère extrêmement contraint par les circonstances liées aux questions de légitimité, de pouvoir effectif, et de place dans l'interaction. Ce sont elles qui prédisposent la construction d'un ethos discursif, qui autorisent ou interdisent, ou du moins régulent, les saillies humoristiques des candidats.

Ainsi le trait d'humour que François Mitterrand se permet en 1988, lors de son débat avec Jacques Chirac, est-il directement informé par une situation d'énonciation et un interdiscours qui balisent ses conditions de production et de réception. À une tirade sans doute mal inspirée de son opposant de droite qui promet un abaissement de la TVA sur la nourriture pour animaux domestiques ${ }^{23}$, Mitterrand rétorque:

Vous n'avez pas le monopole du cœur pour les chiens et les chats, je les aime moi aussi.

28 avril 1988, débat télévisé J. Chirac/F. Mitterrand.

La formule joue sur la dérision, de nature héroï-comique, juxtaposant les termes graves, solennels (" le monopole du cœur») à une référence désacralisante (les chiens et les chats); l'humour, qui apparaît ici doublement voire triplement pertinentisé, conforte l'ethos présidentiel de son auteur. F. Mitterrand saisit la balle au bond pour afficher sa mémoire historique de l'exercice télévisuel, il cite, quatorze ans après, "la remise en place » que lui avait infligée V. Giscard d'Estaing, avec son célèbre "vous n'avez pas le monopole du cœur": ce faisant, 1. il se montre capable d'autodérision, 2. il s'approprie dans la même foulée les mots du vainqueur, 3. sous la boutade, il affiche la posture dominante d'un homme désormais mûri par sa fonction, qui n’a pas oublié une expérience malheureuse, mais qui sait tirer les leçons du passé.

23. Une telle promesse, mal ciblée, ou mal venue, laissait apparaître son caractère dérisoire par rapport aux questions attendues dans un débat de tel enjeu. 
Toujours au crédit de la maîtrise du rapport de place, et dans le même débat, on se rappelle une autre réplique mitterrandienne particulièrement retorse. Jacques Chirac excédé par l'appellatif «monsieur le Premier ministre » dont son interlocuteur le gratifiait, dénonçait sous la pseudocourtoisie insistante, la convocation d'un rapport de place institutionnel, en l'occurrence inégalitaire: ici, protestait-il, le débat devait se dérouler non entre un Président de la République et son chef de gouvernement, mais bien entre deux candidats sur un pied d'égalité:

Ce soir, je ne suis pas le Premier ministre et vous n'êtes pas le Président de la République, nous sommes deux candidats, à égalité, et qui se soumettent au jugement des Français. Le seul qui compte. Vous me permettrez donc de vous appeler Monsieur Mitterrand.

Ibid.

"Vous avez raison ", concède alors Mitterrand, enchaînant aussitôt avec le terrible faux lapsus, "monsieur le Premier Ministre ». Récidive moqueuse fallacieusement couverte par l'excuse de l'habitude, et qui ne fait que confirmer l'ascendant "naturel ", la position d'autorité d'un Président à qui de fait revient le dernier mot. L'effet perlocutoire ratifia cette place puisque Jacques Chirac, décontenancé, resta sans voix.

Le mot d'esprit tend à ridiculiser l'interlocuteur en surjouant la politesse et le respect du code institutionnel. L'humour et l'ironie conjuguent ici leurs effets, articulés par la visée pragmatique du moment: conserver le bénéfice d'un ethos préalable avantageux. Cette interaction nous rappelle le jeu entre ethos préalable et ethos discursif, elle révèle aussi celui qui sépare l'ethos discursif d'un troisième avatar: l'ethos validé.

\subsection{Des enjeux et des rôles}

Comme plusieurs exemples l'ont montré, l'humour conforte à merveille un rapport de place mais, dans l'arène politique, il n'a guère de chance de le renverser.

On a postulé d'une manière générale que la dérision est contraire à l'ethos sérieux attendu d'un responsable politique. Mais un autre arrièreplan plombe implicitement le tableau. En France, la tradition humoristique, souvent représentée par le pamphlet ou la satire, est associée à des courants marginaux, d'extrême-droite ou anarchistes ${ }_{z}$ dont le discours est d'un " mauvais genre " pour un futur Président. L'humour en politique est d'abord le fait de ceux qui brocardent l'ordre établi, sans prétendre proposer de véritable alternative, de programme viable respectueux des institutions. Jouant avec le rapport au vrai, il cultive volontiers le paradoxe. Il apparait alors comme une conduite énonciative de provocation, 
disconvenante sur la durée pour ceux qui voudraient tenir les rênes d'un pays: même si un chef d'État doit entendre la déstabilisation de la doxa véhiculée par l'humoriste, l'humoriste n'a pas vocation à gouverner. La candidature éphémère de Coluche lors de la campagne de 1981 révèle une place intenable ${ }^{24}$.

Il faut donc respecter une logique des rôles. L'humour du candidat président, est sous condition expresse: il doit afficher sa présidentialité, et s'il peut jouer avec son image, c'est sans prendre le risque de l'affaiblir. Le trait d'esprit es qualités humanise l'homme de pouvoir et signe en même temps sa supériorité intellectuelle; en ce sens il récuse la transgression ou la vulgarité.

Certes, on rit volontiers " aux dépens de ", mais l'attaque ad hominem est mal perçue entre républicains qui se respectent. À cet égard l'humour du présidentiable se démarque du sarcasme des outsiders qui stigmatisent l'adversaire et dénoncent à travers lui le système. En politique, il en va de l'humour comme des pratiques discursives en général: les différences tendent à se mesurer entre partis de gouvernement et partis tribunitiens, plus qu'entre discours de gauche et discours de droite (Mayaffre, 2012a). On le voit avec Jean-Marie Le Pen qui cultive un ethos de provocateur et rejette explicitement l'establishment. Ses jeux de mots et autres traits s'adressent exclusivement à son camp, les "petits, les sans-grade ", à qui il offre un exutoire. Cet humour en vase clos, aux antipodes du consensus, rejoint l'" humeur ", voire l'insulte. Son "Durafour crématoire " aux relents négationnistes ${ }^{25}$, ou son "immaculée contraception " à propos de Simone Veil ${ }^{26}$, lui ferment les portes de la République ${ }^{27}$ : Le Pen transgresse jusqu'au discrédit. De l'autre côté de l'échiquier politique, les

24. L'émission à succès de Karl Zéro, Le vrai journal (1996-2006), dans laquelle l'animateur tutoyait les hommes politiques, abordant avec eux des sujets graves sur un ton humoristique a fini par s'épuiser, alors que sur la même chaîne (Canal +), Les Guignols de l'info, qui eux donnent sans équivoque dans le genre burlesque, perdurent sans difficulté. On observe également qu'en dépit de ses dires, Beppe Grillo n’est pas un "Coluche italien ", mais le porte-parole d'une mouvance populiste.

25. Le 2 septembre 1988, Le Pen avait qualifié le ministre Michel Durafour de « Monsieur Durafour-crématoire ». Il fut condamné à 10000 francs d'amende par la cour d'appel de Paris.

26. À l'origine cette expression fut utilisée par le ministre de la santé Jean Foyer, à propos de Lucien Neuwirth, promoteur de la contraception orale en 1967. Mais J.-M. Le Pen significativement la revendique pour Simone Veil accusée avec la loi sur l'IVG de légaliser une extermination: le jeu de mot à connotation antisémite est un insigne d'extrême-droite depuis les années trente.

27. Marine Le Pen, désireuse d'offrir une respectabilité à son parti, a renoncé précisément à ce type d'humour. 
mauvais bons mots sont moins fréquents, mais « le capitaine de pédalo » de Jean-Luc Mélenchon, visant François Hollande, construit à sa manière une marginalité incompatible avec une responsabilité gouvernementale.

\section{Pour conclure}

Interviewé un jour par Anne Sinclair, l'humoriste Guy Bedos disait que l'on pouvait rire de tout, mais que tous les rires n'étaient pas de bonne qualité, et Coluche de son côté affirmait qu'on pouvait plaisanter sur n'importe quel sujet mais pas avec n'importe qui. Ils savaient d'expérience que les conditions de félicité sont complexes pour l'acte de dérision. $A$ fortiori lorsque celui-ci s'invite dans une campagne électorale. François Hollande, candidat à la Présidence de la République en 2012, s'est appliqué à construire l'ethos sérieux requis pour la fonction, il a mis alors sous contrôle son humour légendaire.

Prenant acte de ce changement, nous avons tenté de cerner le «bon usage » de la dérision en politique et singulièrement sa compatibilité avec la figure présidentielle. Il s'avère que contrairement à un sentiment naïf, la partition humoristique ne relève pas du seul choix de l'énonciateur. L'exercice de l'humour est largement prédéterminé par un contexte de production et de réception où interferent la réputation, la légitimité, le pouvoir, les commentaires médiatiques: les places qui se dessinent montrent des recoupements mais aussi des écarts entre ethos préalable et ethos construit, entre ethos construit et ethos validé.

Même pris comme échappatoire, l'humour a des allures de luxe conversationnel qui, dans une interaction verbale, esthétise et conforte une posture dominante, celle du président sortant encore en exercice, et favori des sondages, ou simplement celle du candidat donné gagnant. Sans doute l'impact des sondages est-il difficile à évaluer, les acteurs politiques affectent à leur égard une improbable sérénité, mais les analystes reconnaissent leur influence sur les comportements: dans nos observations, l'usage de l'humour semble réussir à celui qui est "bien placé ": Mitterrand en 1988 ramenant Chirac à son rôle de second, et Hollande en 2012, dont la courbe des sondages favorable ne s'est jamais inversée. Mais cette affinité impose au trait d'esprit une certaine qualité. Les Français, on l'a noté, semblent attachés à l'image d'un monarque républicain: cette image appelle un humour distancé, qui vous élève au-dessus de vous-même sans doute, et des autres à coup sûr.

Cette prise de hauteur de l'humour explique en grande partie ses conditions d'exercice dans le discours politique. Quand il n'est pas le luxe discret de celui qui domine, il devient le luxe ostentatoire de celui 
qui ne peut gagner, et qui de lui-même se met hors-jeu. Mais au-delà de cette détermination générale, l'humour subit ici les contraintes spécifiques d'une élection présidentielle au suffrage universel à deux tours.

Élection uninominale au suffrage universel, elle suppose, dit-on, la rencontre charismatique d'un homme avec les citoyens d'un pays, rencontre qui doit transcender le simple clivage des partis, pour rassembler au-delà de son camp. Il en découle la fameuse nécessité de "fendre l'armure ", de s'ouvrir "dans sa vérité » : pour F. Hollande, et devant un public de sympathisants, vint alors le moment de rappeler son naturel, l'humour, dans le discours du Bourget. Élection à deux tours, elle impose de ferrailler d'abord et de réconcilier ensuite: d'éliminer des concurrents sans interdire leur ralliement. La dérision doit s'adapter à de subtils dosages qui relativisent l'opposition théorique humour vs ironie, comme on l'a vu à l'égard de François Bayrou. Elle doit aussi répondre à des attentes divergentes, flatter l'idéal de pugnacité des uns, l'idéal d'intelligence des autres, et plus encore l'idéal d'une majorité qui voudrait les combiner: le bon usage de l'humour répond à l'exercice d'équilibriste d'un bon ethos de candidat.

\section{Références bibliographiques}

Adam J.-M. ( ), La linguistique textuelle. Des genres de discours aux textes, Paris, Nathan.

Amossy R. (dir) (1999), Images de soi dans le discours, Paris-Lausanne, Delachaux et Nestlé.

- (2006-2 éd.), L’Argumentation dans le discours, Paris, Colin.

- (2010), La présentation de soi. Ethos et identité verbale, Paris, PUF.

Aristote (1991), Rhétorique, Livres I et II, texte établi et traduit par M. Dufour, Paris, Gallimard, coll. « Tel ».

Berrendonner (2002), "Portrait de l'énonciateur en faux naïf ", Figures du discours et ambiguïté (M. Bonhomme dir.), Semen, 15, p. 113-126.

Bonhomme M. (2005), Pragmatique des Figures du discours, Paris, Champion.

- (dir) (2013), Les figures de style vues par la linguistique contemporaine, L'Information grammaticale, 137.

Brown P. \& Levinson S. (1987), Politeness. Some Universals in Language Use, Cambridge, CUP. 
Charaudeau P. (2005), Le discours politique. Les masques du pouvoir, Paris, Vuibert.

- (2006), "Des catégories pour l'humour?", Questions de communication, 10, p. 19-41.

- (2011), "Des catégories pour l'humour. Précisions, rectifications, compléments ", dans M. D. Vivero García (dir.), Humour et crises sociales, Paris, L'Harmattan, p. 9-44.

Charaudeau P. et Maingueneau D. (dir.) (2002), Dictionnaire d'Analyse du discours, Paris, Seuil.

Flahault F. (1979), La Parole intermédiaire, Paris, Seuil.

Guyonnet P. (1997), " Du sacré en politique ", Les Cahiers internationaux de sociologie, 102, p. 161-181.

Hollande F. (2012), Changer de destin, Paris, Laffont.

Jaubert A. (2008), « Dire et plus ou moins dire. Analyse pragmatique de l'euphémisme et de la litote ", Langue française, 160, p. 106-116.

- (2009), "Strates énonciatives et lecture ironique ", dans F. Boissiéras \& M.-H. Servet (dirs), Hiérarchisation énonciation, Louvain-Paris, Peeters (p. 205-219).

- (2011), "Le calembour ou la pragmatique du trait/facile/ ", Les Figures de l'à peu-près (A. Rabatel dir.), Le Français Moderne, 1, p. 33-43.

- (2013), «La figure et le dess(e)in. Les conditions de l'acte ironique ", Les figures de style vues par la linguistique contemporaine (M. Bonhomme dir.), L’information grammaticale, 137, p. 29-35.

Jouan H. (2012), Le petit Hollande illustré par l'exemple, Paris, Le Nouveau monde éditions.

Kerbrat-Orecchioni C. (1986), L’implicite, Paris, Colin.

Maingueneau D. (1999), "Ethos, scénographie, incorporation ", dans R. Amossy (dir.), Images de soi dans le discours, Paris-Lausanne, Delachaux et Nestlé (p. 75-100).

Mayaffre D. (2005), "Analyse du discours politique et Logométrie: point de vue pratique et théorique ", Langage et Société, 114, p. 91121.

- (2012a-rééd.), Le discours présidentiel sous la Ve République. Chirac, Mitterrand, Pompidou, de Gaulle, Paris, Presses de Sciences Po. 
- (2012b), Mesure et démesure du discours. Nicolas Sarkozy 2007-2012, Paris, Presses de Sciences Po.

Rabatel A. (2013), « Humour et sous-énonciation (vs ironie et sur-énonciation) ", Les figures de style vues par la linguistique contemporaine, (M. Bonhomme dir.), L'Information grammaticale, 137, p. 36-42.

Tsakona V. \& Popa D. E. (2012), Studies in Political Humour, Amsterdam/Philadelphia, Benjamins.

Vivero Garcia M. D. (2011), « L'ironie, le sarcasme, l'insolite... peuventils bousculer les valeurs dominantes? ", dans M. D. Vivero García (dir.), Humour et crises sociales, Paris, L'Harmattan (p. 45-56). 